\title{
Eddy correlation measurements of oxygen uptake in deep ocean sediments
}

\author{
Peter Berg ${ }^{1 *}$, Ronnie N. Glud ${ }^{2}$, Andrew Hume ${ }^{1}$, Henrik Stahl², Kazumasa Oguri ${ }^{3}$, Volker Meyer ${ }^{4}$, \\ and Hiroshi Kitazato ${ }^{3}$ \\ ${ }^{1}$ Department of Environmental Sciences, University of Virginia, 291 McCormick Road, Charlottesville, VA 22904-4123, USA \\ ${ }^{2}$ Dunstaffnage Marine Laboratory, Scottish Association of Marine Sciences, Oban, Scotland P-A37 1QA, UK \\ ${ }^{3}$ Institute of Biogeosciences, JAMSTEC, Natsushimacho 2-15, Yokosuka, Kanagawa, 237-0061, Japan \\ ${ }^{4}$ Max Planck Institute for Marine Microbiology, Celsiusstrasse 1, 28359 Bremen, Germany
}

\begin{abstract}
We present and compare small sediment-water fluxes of $\mathrm{O}_{2}$ determined with the eddy correlation technique, with in situ chambers, and from vertical sediment microprofiles at a $1450 \mathrm{~m}$ deep-ocean site in Sagami Bay, Japan. The average $\mathrm{O}_{2}$ uptake for the three approaches, respectively, was $1.62 \pm 0.23$ (SE, $n=7$ ), $1.65 \pm 0.33$ $(n=2)$, and $1.43 \pm 0.15(n=25) \mathrm{mmol} \mathrm{m}^{-2} \mathrm{~d}^{-1}$. The very good agreement between the eddy correlation flux and the chamber flux serves as a new, important validation of the eddy correlation technique. It demonstrates that the eddy correlation instrumentation available today is precise and can resolve accurately even very small benthic $\mathrm{O}_{2}$ fluxes. The correlated fluctuations in vertical velocity and $\mathrm{O}_{2}$ concentration that give the eddy flux had average values of $0.074 \mathrm{~cm} \mathrm{~s}^{-1}$ and $0.049 \mu \mathrm{M}$. The latter represents only $0.08 \%$ of the $59 \mu \mathrm{M}$ mean $\mathrm{O}_{2}$ concentration of the bottom water. Note that these specific fluctuations are average values, and that even smaller variations were recorded and contributed to the eddy flux. Our findings demonstrate that the eddy correlation technique is a highly attractive alternative to traditional flux methods for measuring even very small benthic $\mathrm{O}_{2}$ fluxes.
\end{abstract}

\section{Introduction}

Measuring $\mathrm{O}_{2}$ flux across the sediment-water interface is a widely used approach to assess benthic production and mineralization of organic material in aquatic environments. In addition, many other biogeochemical sediment processes are tightly regulated by $\mathrm{O}_{2}$ exchanged across the sedimentwater interface. Hence, the ability to monitor this flux accurately is very important in understanding the regulation of biogeochemical cycles of various size- and time-scales in the aquatic environment.

*Corresponding author: E-mail: pb8n@virginia.edu

\section{Acknowledgments}

We are grateful to the crew members of the ROV Hyperdolphin and the research vessel R.V. Natushima for outstanding support of our January 2008 cruise during which we recorded the data presented here. We thank Anni Glud for technical assistance and skillful sensor fabrication. The study was supported by grants from the National Science Foundation (OCE-0536431), the Danish National Science Research Council, the EU Commission (GOCE-CT2003-505564), NERC (SOFIgrant, NE/F012691/1), Japan Society for the Promotion of Science (18710021, 17204046), and JAMSTEC.
The in situ methods that have been traditionally used to determine benthic $\mathrm{O}_{2}$ exchange-chamber incubations and interpretations of vertical $\mathrm{O}_{2}$ microprofiles-have limitations and potential problems. Benthic chambers enclose a small sediment area plus a portion of the overlying water, and thus obstruct the natural hydrodynamic flow. In addition, the natural fauna density and behavior may not be well represented within the enclosure. Microprofiles provide detailed depth distributions of $\mathrm{O}_{2}$ at discrete points, but horizontal variability at larger scales may cause flux estimates to be nonrepresentative of the environment. Furthermore, fauna contributions to the $\mathrm{O}_{2}$ flux cannot easily be quantified from microprofiles.

Recently, noninvasive eddy correlation measurements of vertical $\mathrm{O}_{2}$ fluxes across the sediment-water interface were introduced in the aquatic environment (Berg et al. 2003a). Since then, other groups have also begun to use the technique (e.g., Kuwae et al. 2006; McGinnis et al. 2008; Brand et al. 2008) that has several significant advantages over traditional flux methods. Eddy correlation measurements are done under true in situ conditions, with minimal disturbances of the sediment, the ambient light conditions or the boundary layer flow over the seafloor. In addition, eddy correlation measurements 
integrate over a much larger area on the sediment surface than traditional flux methods (Berg et al. 2007).

Given the newness of eddy correlation measurements in the aquatic environment, we have limited experience with the approach and several aspects of the technique still need to be investigated. One key aspect is the precision of eddy correlation fluxes-a question that is often discussed but has not yet been fully answered. Eddy correlation measurements and parallel chamber incubations in carbon-enriched coastal muddy sediments compare well (Berg et al 2003a). Based on these validations, we are confident that larger $\mathrm{O}_{2}$ fluxes $(>\sim 50 \mathrm{mmol}$ $\mathrm{m}^{-2} \mathrm{~d}^{-1}$ ) can be resolved with adequate precision. However, we have not determined whether technical limitations would prevent us from measuring smaller fluxes with acceptable precision and accuracy. Such fluxes put the highest demands on the eddy correlation instruments to resolve the small fluctuations in vertical velocity and $\mathrm{O}_{2}$ concentration that together form the eddy flux signal. Difficulties measuring small fluxes potentially would confine the approach to coastal sediments with high benthic turnover rates, and thus large fluxes.

A review of published in situ benthic $\mathrm{O}_{2}$ uptake measurements suggests that uptake decreases exponentially with water depth (Glud 2008). Sediments at water depths below $250 \mathrm{~m}$ cover $>90 \%$ of the global ocean. Even though they only have $\mathrm{O}_{2}$ uptake rates in the range of 0.5 to $5 \mathrm{mmol} \mathrm{m}^{-2}$ $\mathrm{d}^{-1}$, they account for $>60 \%$ of the global benthic carbon mineralization (Glud 2008). This, together with the common observation that phototrophic $\mathrm{O}_{2}$ production at times can counterbalance respiration processes in coastal shallow waters, makes it important to investigate whether small benthic $\mathrm{O}_{2}$ fluxes also can be measured accurately with the eddy correlation technique.

Identifying experimental setups to examine this potential limitation of the technique was not trivial. First, the measurements had to be performed in the field because realistic in situ conditions cannot be reproduced in a flume. Second, the measurements had to be performed in sediments where other flux methods work well to obtain valid reference data for comparison. Third, we have learned that eddy correlation derived fluxes, at least at shallower sites $(<\sim 30 \mathrm{~m})$, often contain considerable short-term variations, triggered by rapid changes in light and current flow (Berg and Huettel 2008), which complicates comparisons with other flux methods. This all seriously limits the sites where such experiments are feasible.

In this article, we compare $\mathrm{O}_{2}$ fluxes determined simultaneously with the eddy correlation technique, with in situ chambers, and from vertical sediment microprofiles at a deep-ocean margin in Sagami Bay, Japan. Putting aside the logistical challenges of working in the deep ocean, the site is nearly ideal as a test site for the eddy correlation technique and comparisons with other techniques. Previous chamber deployments at this site have shown small $\mathrm{O}_{2}$ uptake with no day-to-day variation (Glud et al. 2009) and the muddy sediment of Sagami Bay is well-suited for chamber deployments and microprofiling.

\section{Methods}

Study site-All data presented here were collected in Sagami Bay, Japan, during a cruise in January 2008. The site is located at $1450 \mathrm{~m}$ water depth in a central part of the bay $\left(35^{\circ} 00.86^{\prime} \mathrm{N}\right.$ $\left.139^{\circ} 21.59^{\prime} \mathrm{E}\right)$ where the fine-grained, muddy sediment has a surface porosity of 0.96 that declines to 0.85 at $\sim 5 \mathrm{~cm}$ depth (Glud et al. 2009) and a total organic carbon content of 3.3\% at the surface that declines to $2.5 \%$ (wet weight; Nakatsuka et al. 2003). Organic matter decay in the water column results in $\mathrm{O}_{2}$-depleted bottom water $(\sim 60 \mu \mathrm{M})$ and an $\mathrm{O}_{2}$ uptake by the sediment that only varies seasonally between $\sim 1$ and $\sim 3 \mathrm{mmol}$ $\mathrm{m}^{-2} \mathrm{~d}^{-1}$ (Glud et al. 2009).

The use of the remotely operated vehicle (ROV) Hyperdolphin and its mother vessel R.V. Natushima (for more information on both vessels, see http://www.jamstec.go.jp/e/about/ equipment/ships/) allowed us to deploy our instruments very near each other with minimal disturbance of the sediment. For further details on the site, see Kitazato et al. (2003), Nakatsuka et al. (2003), Glud et al. (2005), and Glud et al. (2009).

Eddy correlation instrument and deployment-The core of our eddy correlation instrument (Fig. 1) consists of an acoustic doppler velocimeter (ADV) and a fast-responding $\mathrm{O}_{2}$ microelectrode mounted directly on an amplifier. The ADV, a Vector from Nortek AS, Norway, was modified by the manufacturer to interface with the amplifier. In addition to the three velocity components $(x, y, z)$ and the $\mathrm{O}_{2}$ concentration, the ADV records temperature, pressure, position above sediment surface, and an array of secondary variables for data quality control. The ADV can store up to $30 \mathrm{~h}$ of continuous data measured at $64 \mathrm{~Hz}$. The $\mathrm{O}_{2}$ microelectrode was of the Clark-type (Revsbech 1989) with an internal reference and a guard cathode, an outer tip diameter of $\sim 5 \mu \mathrm{m}$, and the ability to capture $90 \%$ of an abrupt change in concentration within $\sim 0.2$ s. The electrode was polarized for at least $10 \mathrm{~h}$ prior to use and calibrated against the known concentration of the bottom water and an electrode reading in anoxic water kept at in situ temperature (Gundersen et al. 1998). The amplifier for the $\mathrm{O}_{2}$ sensor signal was developed by the Max Planck Institute for Marine Microbiology, Germany, specifically for eddy correlation measurements. All components were powered by an external battery and are depth-rated to $2000 \mathrm{~m}$ or more. The three velocity components and the $\mathrm{O}_{2}$ concentration are typically recorded 5 to $30 \mathrm{~cm}$ above the sediment surface and at a rate of $64 \mathrm{~Hz}$. Eight to $16 \mathrm{~Hz}$ data representation is sufficient to describe the full-frequency spectrum carrying the eddy flux signal, but the $64 \mathrm{~Hz}$ data recording allows us to reduce the noise by lumping consecutive recordings.

For shallow water deployments, the eddy correlation components have been mounted on a light frame that can be handled by scuba divers (Berg and Huettel 2008). This frame is designed for minimal disturbance of the natural flow over the seafloor, but logistic constraints made it impossible to use in this study. Alternatively, we attached the eddy correlation components to a 

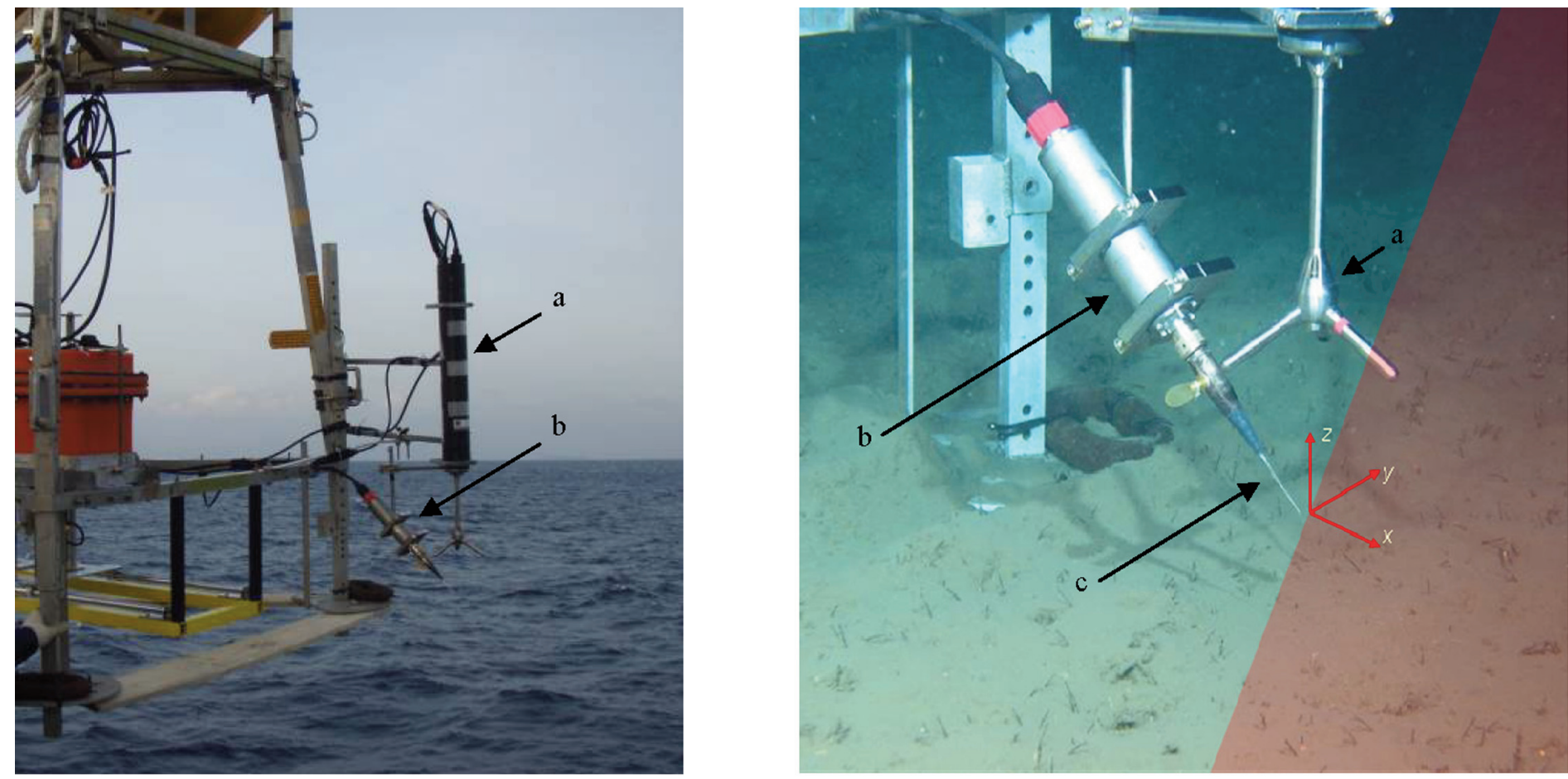

Fig. 1. (A) Eddy correlation instrument attached to the corner of a free-falling sediment-profiling instrument. The bulky structure of this instrument prohibited recordings of undisturbed eddy correlation data for some current directions. (B) Close-up of the eddy correlation instrument in the clear bottom water. Only eddy correlation data with mean current velocities above $1 \mathrm{~cm} \mathrm{~s}^{-1}$ and directed from the red area toward the center of the coordinate system was used to calculate the flux. The eddy correlation instrument consists of (a) an Acoustic Doppler Velocimeter (ADV), (b) an amplifier, and (c) a fast-responding $\mathrm{O}_{2}$ microelectrode. Note the flexible rubber bladder filled with paraffin oil as part of the electrode's pressure compensation.

free-falling sediment-profiling instrument described in more detail below (Fig. 1). At the bottom, the ROV moved and oriented the profiling instrument so that the eddy instrument pointed up into the current. During periods in which the current direction deviated more than $\pm 90^{\circ}$ from its initial direction, however, the bulky structure of the profiling instrument obstructed the natural flow and data recorded during these periods were discarded. Before deployments, the ADV was programmed to measure in 15 min bursts, each consisting of a 14 min continuous data recording at $64 \mathrm{~Hz}$ followed by a $1 \mathrm{~min}$ pause.

Benthic chamber and deployment-The cylindrical transparent benthic chamber with an inner diameter of $19 \mathrm{~cm}$ was deployed and recovered by the ROV, which also provided close-up photos of the installed chamber from which the interior water column height above the enclosed sediment was determined. The overlying water in the chamber was stirred by a rotating disc during deployments and the interior water column concentration of $\mathrm{O}_{2}$ was measured at $0.1 \mathrm{~Hz}$ with two electrodes. These custom-made sensors had an outer tip diameter of $1.5 \mathrm{~mm}$ but a tip opening and membrane diameter of only 2-4 $\mu \mathrm{m}$, which combine to give a rugged design with the advantages of microsensors (i.e., fast response and low stirring sensitivity; Glud et al. 1995). The sensors were calibrated against the known concentration of the bottom water and an electrode reading in anoxic water kept at in situ temperature (Gundersen et al. 1998).

Microprofiling instrument and deployment-Microprofiles were measured in the sediment with a free-falling profiling instrument (Glud et al. 2009) outfitted with microelectrodes for measuring $\mathrm{NO}_{3}^{-}$, resistivity, and $\mathrm{O}_{2}$. Only the $\mathrm{O}_{2}$ data are presented here. The profiling instrument, also with the eddy correlation components attached, was lowered below the water surface from R.V. Natushima and released for the descent to the bottom. A set of top-mounted floats kept the instrument upright, and their buoyancy was balanced carefully to give a sinking speed of $\sim 25 \mathrm{~m} \mathrm{~min}^{-1}$. At the bottom, the ROV moved the instrument to a location that was not impacted by the landing and then activated the computer controlling the profiling procedure. After activation, the array of microsensors was moved vertically toward and into the sediment in increments of $200 \mu \mathrm{m}$ and after a total traveled distance of $7 \mathrm{~cm}$, the sensor array was moved back to its original position. The array was then moved $12 \mathrm{~mm}$ horizontally to measure a new series of microprofiles. All $\mathrm{O}_{2}$ profiles included two inherent calibration points; one recorded in the bottom water and one below in the anoxic sediment layer below $\mathrm{O}_{2}$ penetration. 
The $\mathrm{O}_{2}$ electrodes used in all three in situ instruments were pressure-compensated using paraffin oil. A small oil-filled flexible rubber bladder connected to the each electrode (Fig. 1) ensured that any small air bubbles or any compression of the electrolyte would not cause the electrode to leak or implode (Reimers and Glud 2000).

\section{Results}

Based on the ROV's positioning system recordings, all measurements were made within a horizontal distance of 40-70 m.

Eddy correlation data and flux extractions-The eddy correlation instrument was deployed twice during the cruise, each time for approximately $30 \mathrm{~h}$. Whereas the $\mathrm{O}_{2}$ microelectrode imploded due to faulty pressure compensation during the first deployment, the second deployment was successful. The 14 min mean current velocity, recorded $10 \mathrm{~cm}$ above the seafloor, varied between 0.1 and $3.4 \mathrm{~cm} \mathrm{~s}^{-1}$ with a mean of $1.1 \mathrm{~cm} \mathrm{~s}^{-1}$. This variation was overlaid by several $\pm 180^{\circ}$ directional changes in current. To ensure an undisturbed and strong eddy flux signal in the data used for flux extraction, each burst was examined carefully and discarded if the mean current velocity was below $1 \mathrm{~cm} \mathrm{~s}^{-1}$ or if the current was not directed from the area marked in red toward the center of the coordinate system in Fig. 1. These requirements reduced the $30 \mathrm{~h}$ long deployment to 7 bursts.

During the cruise, we used a newly manufactured ADV that, because of faulty electric wiring, mistakenly stopped polarizing the $\mathrm{O}_{2}$ microelectrode during the one min pause at the end of each 15 min burst. This mistake caused the initial $\mathrm{O}_{2}$ sensor readings in each burst to be contaminated with substantial noise. As a result, the first $0.5 \mathrm{~min}$ of each burst was discarded. This strict data evaluation left us with $94.5 \mathrm{~min}$ of high quality data, or 7 bursts, each with 13.5 min long $64 \mathrm{~Hz}$ data sequences.

The $\mathrm{O}_{2}$ flux extraction was completed in several steps. First, the $64 \mathrm{~Hz}$ data, consisting of three velocity components $(x, y$, $z$ ) and one $\mathrm{O}_{2}$ concentration for each point in time, were lumped by averaging every 8 data points to give less noisy 8 $\mathrm{Hz}$ data. An example of such $8 \mathrm{~Hz}$ data in three consecutive bursts is shown in Fig. 2, together with the recorded measuring height above the seafloor.

The vertical eddy flux for each burst is defined as $\overline{u_{z}^{\prime} C^{\prime}}$ where $u_{z}^{\prime}$ is the fluctuating vertical velocity away from its mean, $C^{\prime}$ is the fluctuating concentration away from its mean, and the bar symbolizes a simple averaging over the burst (Berg et al. 2003a). Several different definitions of the mean vertical velocity and mean concentration are commonly used in eddy flux extractions (Berg et al. 2003a). For this study, we defined these means as least-square linear fits to the $8 \mathrm{~Hz}$ data in each burst. Referring to these linear fits as $\bar{u}_{z L}$ and $\bar{C}_{L}$, values of $u_{z}^{\prime}$ and $C^{\prime}$ were then calculated as $u_{z}-\bar{u}_{z L}$ and $C-\bar{C}_{L}$ where $u_{z}$ and $C$ represent the $8 \mathrm{~Hz}$ data. With known time series of $u_{z}^{\prime}$ and $C^{\prime}$, the flux was calculated for each burst together with the associated cumulative flux (Fig. 2). The three $\mathrm{O}_{2}$ fluxes shown were $-2.46,-2.15$, and $-1.57 \mathrm{mmol} \mathrm{m}^{-2} \mathrm{~d}^{-1}$, and the average of all 7 flux estimates extracted from the acceptable bursts in this deployment was $-1.62 \pm 0.23$ (SE) $\mathrm{mmol} \mathrm{m}^{-2} \mathrm{~d}^{-1}$.

This flux extraction was executed in the time domain, but it also can be performed in the frequency domain after Fast Fourier transformations of $u_{z}^{\prime}$ and $C^{\prime}$. In that case, the co-spectrum of $u_{z}^{\prime}$ and $C^{\prime}$ expresses the flux contribution as a function of the eddy frequency (Stull 1988; Berg et al. 2003a). This makes the co-spectrum a valuable quantity when evaluating the response time of sensors for use in eddy correlation measurement. The co-spectrum generally contains considerable noise. As a result, it is cumulated from higher toward lower frequencies. An example of a normalized cumulative co-spectrum for one of the bursts is shown in Fig. 3.

Benthic chamber data and flux extractions-The benthic chamber was deployed twice during the cruise, each time for approximately $24 \mathrm{~h}$. The $\mathrm{O}_{2}$ concentration recorded in the chamber is shown in Fig. 4. For each deployment, one $\mathrm{O}_{2}$ flux value was derived from a linear fit to the decreasing $\mathrm{O}_{2}$ concentration measured during incubation in the stirred body of water within the chamber (Fig. 4). For the two deployments, 21.1 and $17.6 \mathrm{~h}$ of data were used to generate these fits, which have $R^{2}$ values of 0.99 and 0.98 and contain concentration drops of 13 and $6.9 \mu \mathrm{M}$, equivalent to $22 \%$ and $12 \%$ of the initial bottom water concentration. With interior water column heights in the chamber of 13.4 and $14.0 \mathrm{~cm}, \mathrm{O}_{2}$ fluxes were estimated to be -1.98 and $-1.31 \mathrm{mmol} \mathrm{m}^{-2} \mathrm{~d}^{-1}$ with an average of $-1.65 \pm 0.33$ (SE) $\mathrm{mmol} \mathrm{m}^{-2} \mathrm{~d}^{-1}$.

Microprofile data and flux extractions-The microprofiling instrument was deployed twice during the cruise and a total of $25 \mathrm{O}_{2}$ profiles were recorded. The two sets of profiles from these deployments were similar, but with substantial differences in a profile-to-profile comparison. Seven representative profiles with respect to observed variations in penetration depth and shape are shown in Fig. 5.

The $\mathrm{O}_{2}$ flux was determined from each profile using Fick's First Law of Diffusion as $-D \times(d C / d x)$, where $D$ is the molecular diffusivity of $\mathrm{O}_{2}$ in the bottom water and $d C / d x$ is the concentration gradient in the diffusive boundary layer (Jørgensen and Revsbech 1985). After locating the position of the sediment-water interface by a close visual inspection of each profile, the gradient $d C / d x$ was determined through least-square linear fitting (Fig. 5). With $D$ equal to $1.17 \times 10^{-5} \mathrm{~cm}^{2} \mathrm{~s}^{-1}$ (Broecker and Peng 1974), the average flux for the 25 profiles was $-1.43 \pm 0.15(\mathrm{SE}) \mathrm{mmol} \mathrm{m}^{-2} \mathrm{~d}^{-1}$.

An alternative way to estimate the flux from an $\mathrm{O}_{2}$ profile is to apply the sediment profile interpretation procedure PROFILE (Berg et al. 1998). This generates a statistically sound fit to the profile and calculates the $\mathrm{O}_{2}$ flux and $\mathrm{O}_{2}$ consumption rates as a function of sediment depth. PROFILE requires information on sediment porosity, and in addition, specification of animal-mediated transport if that is deemed important. Assuming here that molecular diffusion was the dominant transport process, the 7 representative profiles (Fig. 5) were fit 

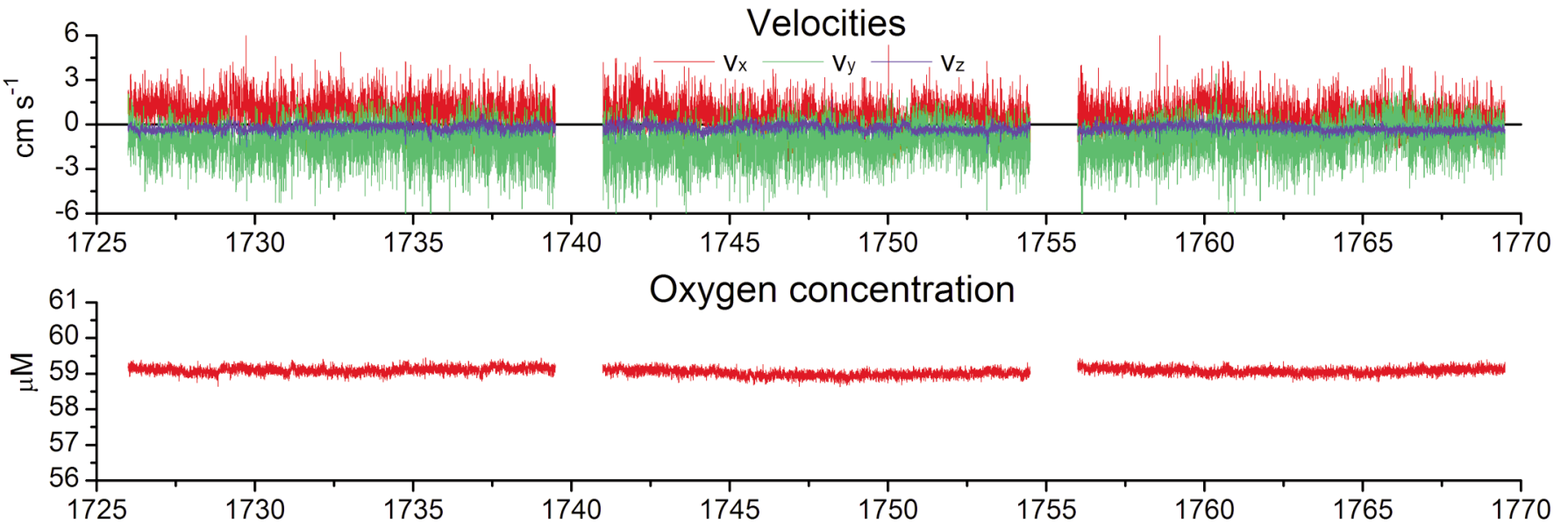

Oxygen concentration
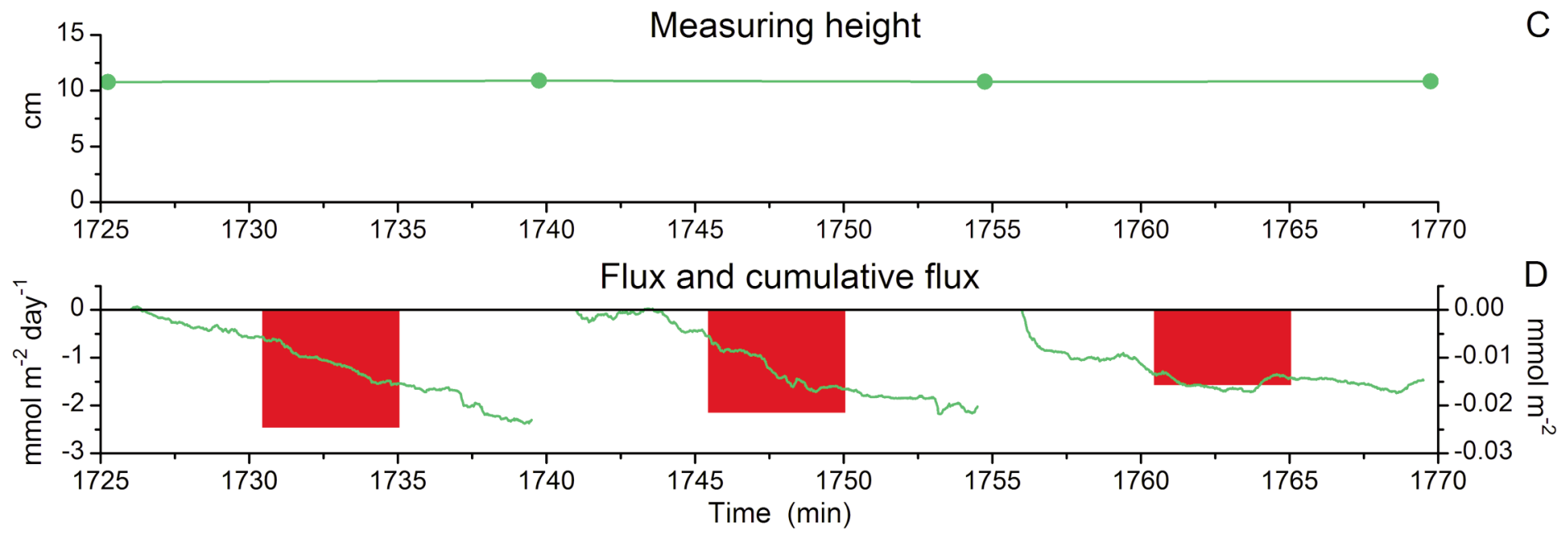

Fig. 2. Eddy correlation data for three consecutive 13.5 min bursts. (A) Raw $8 \mathrm{~Hz}$ velocity data $(x, y, z)$. The visible high-frequency noise arose from the low particle concentration in the bottom water. (B) Raw $8 \mathrm{~Hz} \mathrm{O}_{2}$ concentration data. (C) Measuring height for velocities and $\mathrm{O}_{2}$ concentration above the sediment surface recorded by the ADV at the beginning and end of each burst. (D) Derived $\mathrm{O}_{2}$ flux for each burst (bars) and the associated cumulative flux (line).

well by PROFILE with $R^{2}$ values of 0.99 or higher. Although some deviation was found for individual profiles between fluxes estimated with the two fitting approaches (Fig. 5), the two means agreed to within $8 \%$, a difference that was not statistically significant.

The average $\mathrm{O}_{2}$ uptake rates determined with in situ chambers, with the eddy correlation technique, and from vertical sediment profiles are shown in Fig. 6. None of these fluxes were significantly different from each other, and they compared well with relative values of 1.00:0.98:0.87.

\section{Discussion}

Eddy correlation fluxes-The concentration of suspended particles at the measuring height of $10 \mathrm{~cm}$ above the bottom (Fig. 2) was low relative to what is usually found in coastal waters. Because the three velocity components (Fig. 2) are derived from the acoustic backscatter of suspended particles, they contain high-frequency noise levels that exceed values from typical coastal settings. If high-frequency noise is defined as the difference between two adjacent $8 \mathrm{~Hz}$ data points, the noise in the vertical velocity had a standard error of $0.17 \mathrm{~cm} \mathrm{~s}^{-1}$. The equivalent number for the $\mathrm{O}_{2}$ concentration was $0.12 \mu \mathrm{M}$. These noise levels exceeded the actual fluctuations in both vertical velocity and $\mathrm{O}_{2}$ concentration that give the eddy flux (see below), but since the noise was randomly distributed around the means (data not shown), noise did not compromise the flux calculation.

The cumulative flux (Fig. 2) is a useful variable when evaluating eddy correlation data and derived fluxes. If the cumulative flux shows a clear linear trend within a burst, it indicates quasisteady state conditions with a constant $\mathrm{O}_{2}$ flux that is equal to the slope of this trend. Further, a clear linear trend indicates a statistically good representation of all eddy sizes that contribute to the flux. On that background, the cumulative flux for the first burst shown in Fig. 2 is a close-to-perfect data sequence with a "clean" and strong eddy flux signal. The cumulative flux for the second and third burst also shows clear trends but with a somewhat uneven progress in the beginning of the bursts, 


\section{Normalized cumulative co-spectrum}

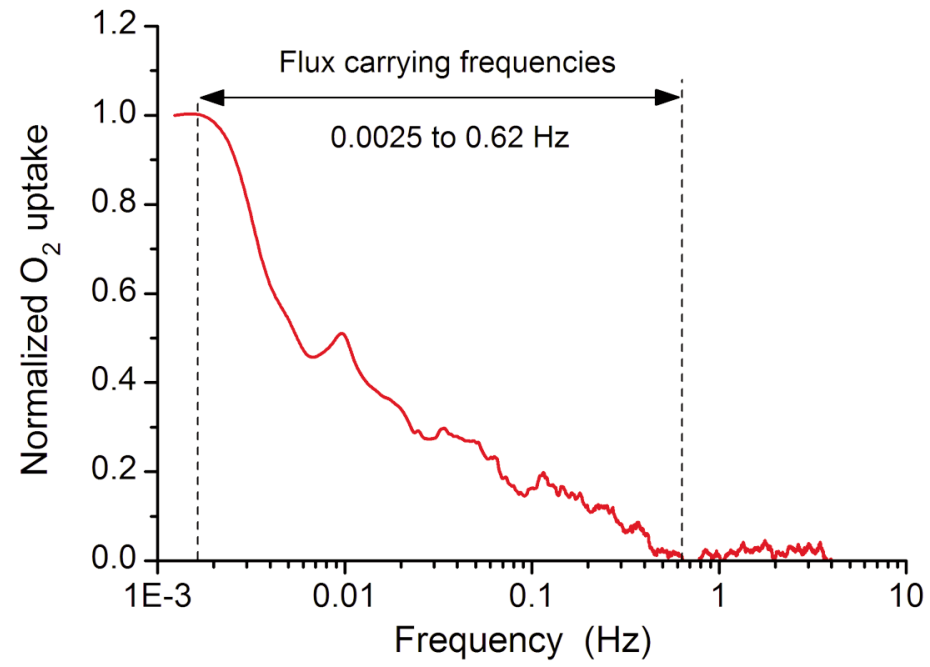

Fig. 3. Normalized cumulative co-spectrum for one representative burst. The flux contributing eddies are found in the 0.62 to $0.0025 \mathrm{~Hz}$ frequency band.

which presumably represented larger eddies. Had longer burst periods been used, they likely would have contained several of these larger eddies and thus resulted in smaller error estimates for the averaged eddy flux than is reported here (Fig. 6).

An apparent technical challenge in eddy correlation measurements is resolving the small fluctuations in vertical velocity $\left(u_{z}^{\prime}\right)$ and $\mathrm{O}_{2}$ concentration $\left(C^{\prime}\right)$. For the latter, the fluctuations occur on top of a high background concentration, which complicates the task further. Obviously, a small flux is characterized by small fluctuations, making small fluxes the most challenging to measure. The size of these fluctuations can be realized by examining the fluctuations of $u_{z}^{\prime}$ and $C^{\prime}$ in the frequency domain. The shape of the normalized cumulative cospectrum (Fig. 3) reveals that all eddies contributing to the flux were found in the 0.62 to $0.0025 \mathrm{~Hz}$ frequency band where this normalized spectrum climbs from 0 to 1 . This band excluded the high-frequency noise visible in the $8 \mathrm{~Hz}$ raw data (Fig. 2). The average absolute value of $u_{z}^{\prime}$ in the 0.62 to $0.0025 \mathrm{~Hz}$ frequency band was $0.078 \mathrm{~cm} \mathrm{~s}^{-1}$ (equivalent to the average absolute deviation of the vertical velocity from its mean). The equivalent value for $C^{\prime}$ was $0.049 \mu \mathrm{M}$. For the latter, this small average fluctuation represented only $0.08 \%$ of the $59 \mu \mathrm{M}$ mean $\mathrm{O}_{2}$ concentration. Further, these characteristic values for $u_{z}^{\prime}$ and $C^{\prime}$ represent averages, which implies that even smaller fluctuations are recorded as flux-contributing eddies. Obviously, this "ultra-high" precision can only be achieved because the fluctuations are relative measurements, and the precision exceeds what can be obtained on the measured absolute values.

Benthic chamber fluxes-The decline in $\mathrm{O}_{2}$ concentration during the two chamber deployments was described very well by the simple linear fits with $R^{2}$ values of 0.99 and 0.98 (Fig. 4). It could be argued that more complex nonlinear
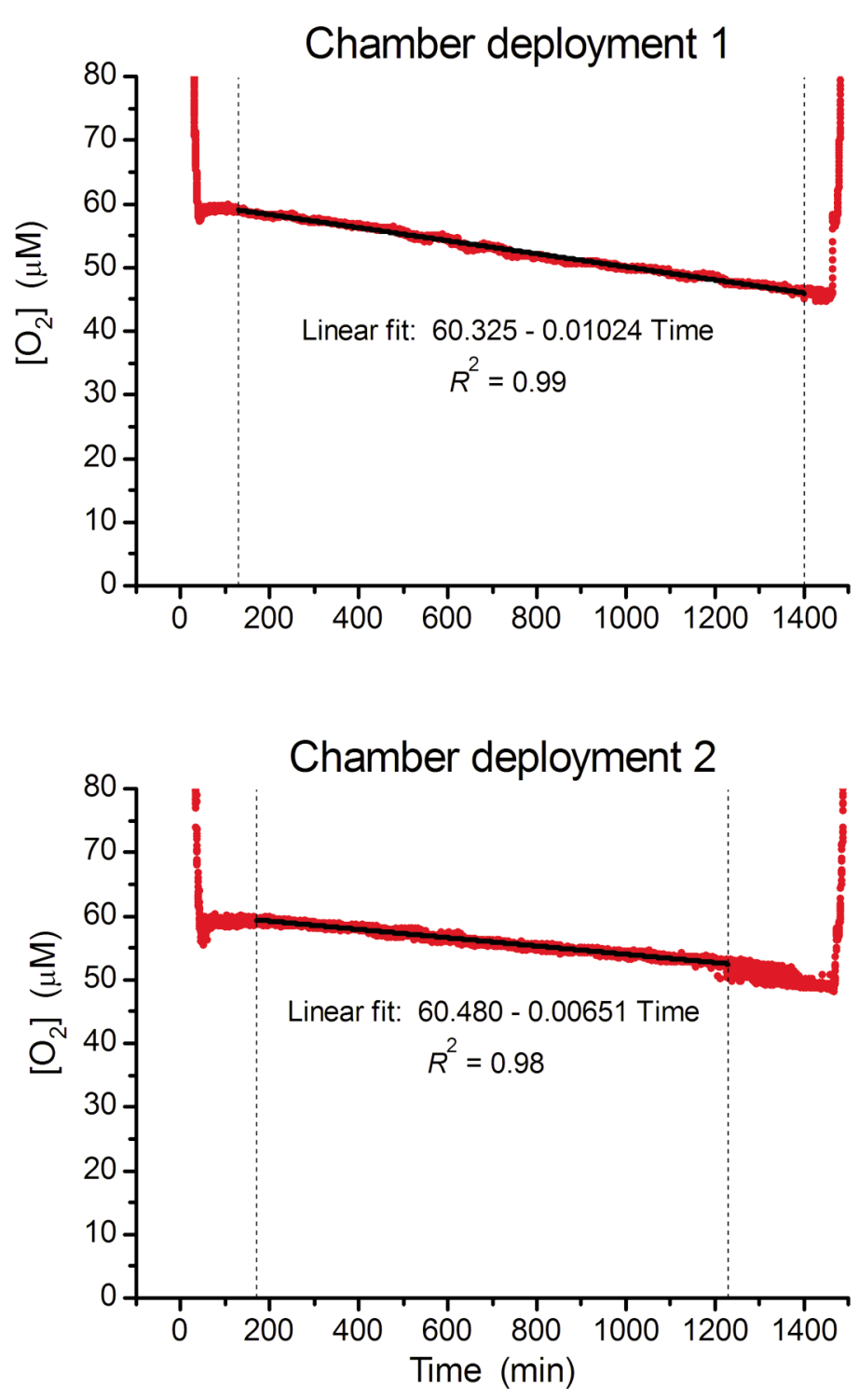

Fig. 4. $\mathrm{O}_{2}$ concentration (dots) in the chamber as measured during its final descent to the seafloor, when it was moved horizontally by the ROV, during the actual incubation, and during its initial recovery. The linear fits (lines), covering 21.1 and $17.6 \mathrm{~h}$ periods, were used for flux extractions.

fitting functions should be used because of the expected changes in the $\mathrm{O}_{2}$ porewater profile during chamber incubation (Devol 1987) and the nonlinear regulations used in many diagenetic models to describe $\mathrm{O}_{2}$ consuming processes (Van Cappellen and Wang 1996; Berg et al. 2003b). However, most often and certainly in this case, they cannot be justified from a statistical point of view as giving a better description of the data. Given the high reproducibility of the simple linear fits, we believe the two chamber fluxes of -1.98 and -1.31 $\mathrm{mmol} \mathrm{m} \mathrm{m}^{-2} \mathrm{~d}^{-1}$ are high-quality estimates. In that light, the relatively large difference of $41 \%$ between the fluxes must reflect real spatial variations of a size-scale equal to or larger than the $19 \mathrm{~cm}$-diameter chamber. 

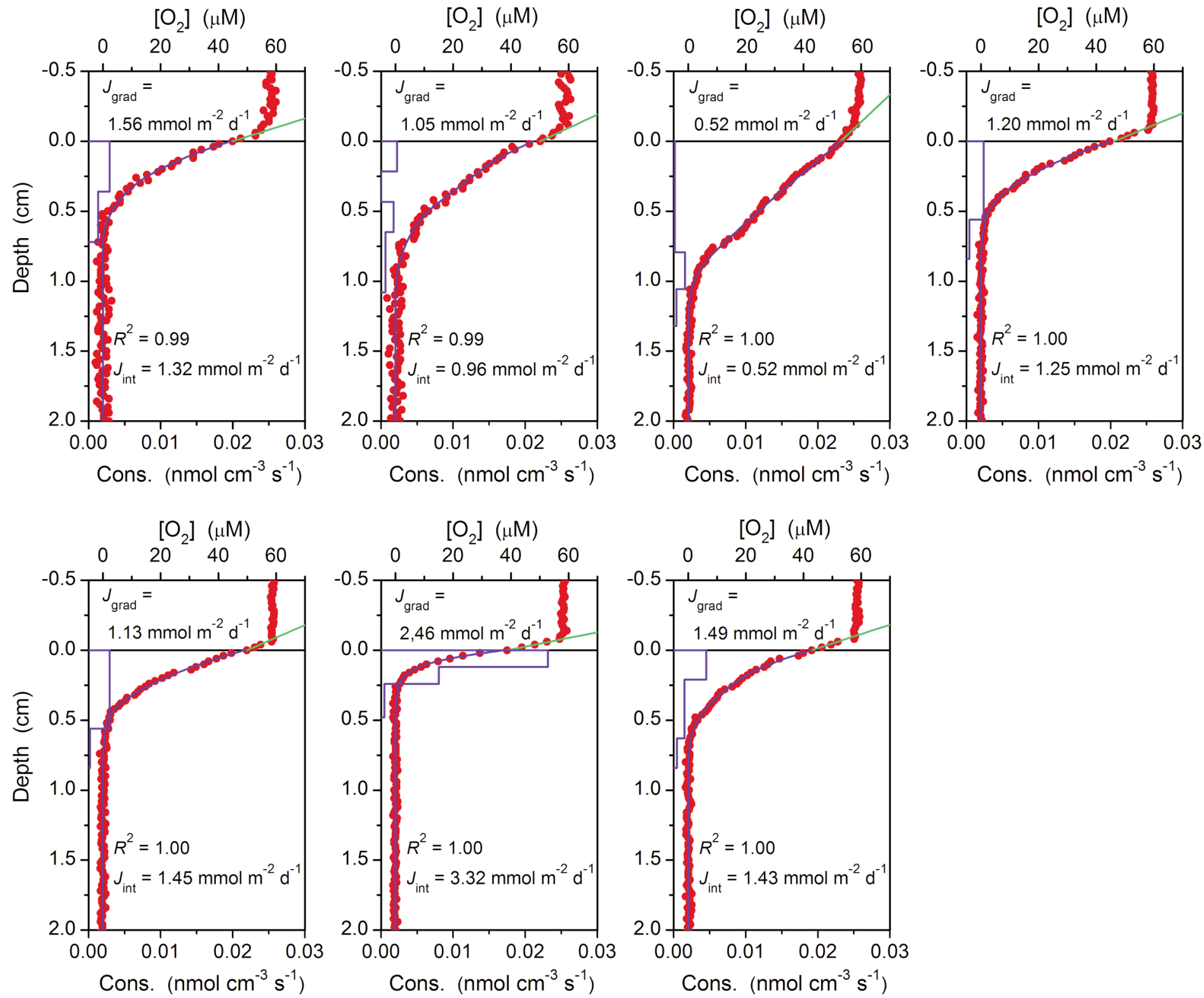

Fig. 5. Seven representative $\mathrm{O}_{2}$ microprofiles (dots) with penetration depth varying from 0.26 to $1.20 \mathrm{~cm}$. The gradients in the diffusive boundary layer (green lines) were used to estimate the $\mathrm{O}_{2}$ flux. The profile interpretation software PROFILE (Berg et al. 1998) was used to produce fits to the profiles (blue lines), $\mathrm{O}_{2}$ consumption rates as a function of depth (blue step curves), and alternative flux estimates. Across the 7 profiles, the means of the two fitting methodologies agree to within $8 \%$.

Fluxes from microprofiles-The measured $\mathrm{O}_{2}$ microprofiles exhibited substantial small-scale spatial variation in the distribution and consumption of $\mathrm{O}_{2}$. This is reflected in the 7 representative profiles shown in Fig. 5, with penetration depths varying between 0.26 to $1.20 \mathrm{~cm}$, and fluxes derived from the profile gradient in the diffusive boundary layer varying between 0.52 and $2.46 \mathrm{mmol} \mathrm{m}^{-2} \mathrm{~d}^{-1}$. Both these variations exceeded a factor of 4 . The fluxes derived with the profile interpretation procedure, PROFILE (Berg et al. 1998), showed a similar pronounced variation. The divergence at this site agrees with previous findings where substantial small-scale variations were resolved with in situ planar optodes (Glud et al. 2005) and in a series of in situ microprofiles measured along $25 \mathrm{~cm}$ long horizontal transects (Glud et al 2009). Glud and coworkers documented that this variation was caused by millimeter-to-centimeter scaled patchiness in high- $\mathrm{O}_{2}$-consuming microenvironments. Previously documented variations in sediment surface topography (Jørgensen and Des Marais 1990) added to this complex pattern and the combined result was a pronounced three-dimensionality in both $\mathrm{O}_{2}$ consumption and $\mathrm{O}_{2}$ concentration. One-dimensional flux estimates, based on either $\mathrm{O}_{2}$ profile fitting in the diffusive boundary layer or with 


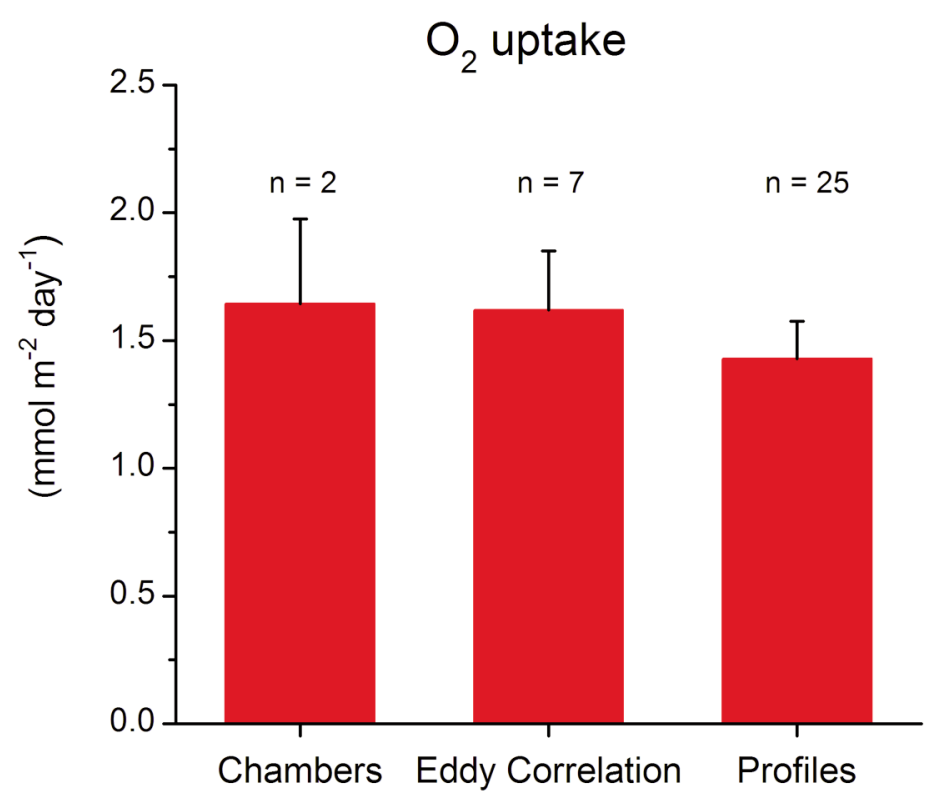

Fig. 6. Average $\mathrm{O}_{2}$ fluxes determined with in situ chambers, with the eddy correlation technique, and from vertical sediment profiles (error bars represent $+1 \mathrm{SE}$ ).

the profile interpretation procedure PROFILE (Berg et al. 1998), should for that reason be interpreted cautiously. This is reinforced by the fact that both fitting methodologies tend to underestimate or exclude the effects of larger animals. Nevertheless, both methods are widely used and accepted, and they give valuable insights into processes that transport and consume (or produce) $\mathrm{O}_{2}$ in sediments.

The average $\mathrm{O}_{2}$ flux for all 25 profiles, calculated from the gradient in the diffusive boundary layer, is not significantly different from the flux measured in the benthic chamber (Fig. 6). This indicates that animal-mediated $\mathrm{O}_{2}$ transport and $\mathrm{O}_{2}$ consumption are of minor importance at this site, a finding that is somewhat atypical. Although the fauna-mediated contribution to the total benthic $\mathrm{O}_{2}$ uptake has been shown to decline with water depth, it usually amounts to $\sim 30 \%$ of the total uptake in the range of $1-1.5 \mathrm{~km}$ water depth (Glud 2008).

Intuitively, deep-ocean sediments are expected to be more homogeneous than sediments at shallower sites. In that light, the distinct millimeter-to-centimeter scaled patchiness in high- $\mathrm{O}_{2}$-consuming microenvironments documented for this site (Glud et al. 2005, 2009), combined with the larger-scaled variations found here in the different chamber deployments, was surprising. Although these data are limited, they suggest that pronounced biogeochemical variations on several sizescales exist in the upper layers of this sediment. We speculate that at least the fine-scale patchiness is caused by depositing nonirrigating infauna (close-up video recordings support this) combined with sediment inputs to the site in the form of marine snow parcels (Glud et al. 2009) that induce short-lived microbial hot spots.
Comparison of eddy correlation fluxes and benthic chamber fluxes-The excellent agreement between our eddy correlation fluxes and the chamber fluxes (Fig. 6) was a key finding of this study. It documents that the eddy correlation instrumentation available today is precise and allows even small fluxes to be resolved with acceptable precision and accuracy. This was unknown until now because earlier validations of the technique (Berg et al. 2003a) focused exclusively on larger fluxes (> 50 $\mathrm{mmol} \mathrm{m}^{-2} \mathrm{~d}^{-1}$ ).

The significance of this result is underscored by the fact that $\mathrm{O}_{2}$ fluxes on the order of $1 \mathrm{mmol} \mathrm{m}^{-2} \mathrm{~d}^{-1}$ dominate in the global ocean (Glud 2008) and are common in coastal shallow waters where phototrophic $\mathrm{O}_{2}$ production can counterbalance respiration processes intermittently. These results are also promising for future applications of the eddy correlation technique for determining benthic fluxes of other solutes for which microsensors exist, or can be developed, and for which it is known that the sediment-water flux is markedly smaller than that of $\mathrm{O}_{2}$.

\section{References}

Berg, P., H. Røy, F. Janssen, V. Meyer, B. B. Jørgensen, M. Hüttel, and D. de Beer. 2003a. Oxygen uptake by aquatic sediments measured with a novel non-invasive eddy correlation technique. Mar. Ecol. Prog. Ser. 261:75-83.

— - , and P. L. Wiberg. 2007. Eddy correlation flux measurements - the sediment surface area that contributes to the flux. Limnol. Oceanogr. 52:1672-1684.

— S. Rysgaard, and B. Thamdrup. 2003b. Dynamic modeling of early diagenesis and nutrient cycling. A case study in an Arctic marine sediment. Amer. J. Sci. 303:905-955.

—, N. Risgaard-Petersen, and S. Rysgaard. 1998. Interpretation of measured concentration profiles in sediment pore water. Limnol. Oceanogr. 43:1500-1510.

— and M. Huettel. 2008. Monitoring the seafloor using the non-invasive eddy correlation technique: integrated benthic exchange dynamics. Oceanography 21:164-167.

Brand, A., D. F. McGinnis, B. Wehrli, and A. Wüest. 2008. Intermittent oxygen flux from the interior into the bottom boundary of lakes as observed by eddy correlation. Limnol. Oceanogr. 53:1997-2006.

Broecker, W. S., and T. -H. Peng. 1974. Gas exchange rates between air and sea. Tellus 26:21-35.

Devol, A. H. 1987. Verification of flux measurements made with in situ benthic chambers. Deep-Sea Res. 34:1007-1026.

Glud, R. N. 2008. Oxygen dynamics of marine sediments. Mar. Biol. Res. 4:243-289.

- J. K. Gundersen, N. P. Revsbech, B. B. Jørgensen, and M. Hüttel. 1995. Calibration and performance of the stirred flux chamber from the benthic lander Elinor. Deep-Sea Res I 42:1029-1042.

— , F. Wenzhöfer, A. Tengberg, M. Middelboe, K. Oguri, and H. Kitazato. 2005.

Distribution of oxygen in surface sediments from central 
Sagami Bay, Japan: In situ measurements by microelectrodes and planar optodes. Deep-Sea Res. I 52:1974-1987.

, H. Stahl, P. Berg, F. Wenzhöfer, K. Oguri, and H. Kitazato. 2009. In situ microscale variation in distribution and consumption of $\mathrm{O}_{2}$ : A case study from a deep ocean margin sediment (Sagami Bay, Japan). Limnol. Oceanogr. 54:1-12.

Gundersen, J. K., N. B. Ramsing, and R. N. Glud. 1998. Predicting the signal of oxygen microsensors from physical dimensions, temperature, salinity, and oxygen concentrations. Limnol. Oceanogr. 43:1932-1937.

Jørgensen, B. B. and N. P. Revsbech. 1985. Diffusive boundary layers and the oxygen uptake of sediments and detritus. Limnol. Oceanogr. 30:111-122.

and D. J. Des Marais. 1990. The diffusive boundary layer of sediments: Oxygen microgradients over a microbial mat. Limnol. Oceanogr. 35:1343-1355.

Kitazato, H., and others. 2003. Long-term monitoring of sedimentary processes in the central part of Sagami Bay, Japan: rationale, logistics and overview of results. Prog. Oceanogr. 57:3-16.

Kuwae, T., K. Kamio, T. Inoue, E. Miyoshi, and Y. Uchiyama. 2006. Oxygen exchange flux between sediment and water in an intertidal sandflat, measured in situ by the eddy-correlation method. Mar. Ecol. Prog. Ser. 307:59-68.
McGinnis, D. F., P. Berg, A. Brand, C. Lorrai, T. J. Edmonds, and A. Wüest. 2008. Measurements of eddy correlation oxygen fluxes in shallow freshwaters: Towards routine applications and analysis. Geophys. Res. Lett. 35:L04403.

Nakatsuka, T., T. Masuzawa, J. Kanda, H. Kitazato, Y. Shirayama, M. Shimanaga, and A. Yamaoka. 2003. Particle dynamics in the deep water column of Sagami Bay, JapanI: Origins of apparent flux of sinking particles. Prog. Ooceanogr. 57:31-45.

Reimers, C. E., and R. N. Glud. 2000. In situ chemical sensor measurements at the sediment-water interface, p. 249-282. In M. S. Varney [ed.], Chemical sensors in oceanography. Gordon and Breach Science Publishers.

Revsbech, N. P. 1989. An oxygen microelectrode with a guard cathode. Limnol. Oceanogr. 34:474-478.

Stull, R. B. 1988. An introduction to boundary layer meteorology. Kluwer Academic Publishers.

Van Cappellen, P., and Y. Wang. 1996. Cycling of iron and manganese in surface sediments: A general theory for the coupled transport and reaction of carbon, oxygen, nitrogen, sulfur, iron, and manganese. Amer. J. Sci. 296:197-243.

Submitted 08 February 2009

Revised 10 June 2009

Accepted 17 June 2009 